Leukemia (2019) 33:2324-2330

https://doi.org/10.1038/s41375-019-0452-6

Multiple myeloma gammopathies

\title{
Exome sequencing identifies germline variants in DIS3 in familial multiple myeloma
}

Maroulio Pertesi ${ }^{1,2} \cdot$ Maxime Vallée $^{1} \cdot$ Xiaomu Wei ${ }^{3} \cdot$ Maria V. Revuelta $\mathbb{D}^{4} \cdot$ Perrine Galia $^{5,6} \cdot$ Delphine Demangel ${ }^{5,6}$. Javier Oliver ${ }^{1,7} \cdot$ Matthieu Foll $^{1} \cdot$ Siwei Chen $^{3} \cdot$ Emeline Perrial $^{8,9} \cdot$ Laurent Garderet $^{10,11,12} \cdot$ Jill Corre $^{13}$. Xavier Leleu ${ }^{14}$ - Eileen M. Boyle ${ }^{15}$. Olivier Decaux ${ }^{16,17,18}$. Philippe Rodon ${ }^{19} \cdot$ Brigitte Kolb $^{20}$ - Borhane Slama ${ }^{21}$. Philippe Mineur ${ }^{22}$ - Eric Voog ${ }^{23}$ - Catherine Le Bris $^{24}$ - Jean Fontan ${ }^{25}$ - Michel Maigre ${ }^{26}$ - Marie Beaumont ${ }^{27}$. Isabelle Azais ${ }^{28}$ - Hagay Sobol ${ }^{29}$ - Marguerite Vignon ${ }^{30}$ - Bruno Royer ${ }^{30}$ - Aurore Perrot ${ }^{31}$ - Jean-Gabriel Fuzibet ${ }^{32}$. Véronique Dorvaux ${ }^{33} \cdot$ Bruno Anglaret $^{34}$. Pascale Cony-Makhoul ${ }^{35}$. Christian Berthou ${ }^{36}$. Florence Desquesnes ${ }^{37}$. Brigitte Pegourie $^{38} \cdot$ Serge Leyvraz ${ }^{39} \cdot$ Laurent Mosser $^{40} \cdot$ Nicole Frenkiel $^{41} \cdot$ Karine Augeul-Meunier $^{42}$. Isabelle Leduc ${ }^{43}$. Cécile Leyronnas ${ }^{44} \cdot$ Laurent Voillat $^{45} \cdot$ Philippe Casassus $^{46} \cdot$ Claire Mathiot $^{47} \cdot$ Nathalie Cheron $^{48}$. Etienne Paubelle ${ }^{49} \cdot$ Philippe Moreau $^{50} \cdot$ Yves-Jean Bignon $^{51} \cdot$ Bertrand Joly $^{52} \cdot$ Pascal Bourquard $^{53} \cdot$ Denis Caillot $^{54}$. Hervé Naman ${ }^{55}$. Sophie Rigaudeau ${ }^{56} \cdot$ Gérald Marit $^{57} \cdot$ Margaret Macro $^{58} \cdot$ Isabelle Lambrecht $^{59}$. Manuel Cliquennois $\mathbb{1}^{60} \cdot$ Laure Vincent $\mathbb{1}^{61} \cdot$ Philippe Helias $^{62} \cdot$ Hervé Avet-Loiseau ${ }^{63} \cdot$ Victor Moreno $\mathbb{1}^{64,65}$. Rui Manuel Reis ${ }^{66,67} \cdot$ Judit Varkonyi $^{68} \cdot$ Marcin Kruszewski $^{69} \cdot$ Annette Juul Vangsted $^{70} \cdot$ Artur Jurczyszyn $^{71}$. Jan Maciej Zaucha ${ }^{72} \cdot$ Juan Sainz $\mathbb{D}^{73} \cdot$ Malgorzata Krawczyk-Kulis ${ }^{74} \cdot$ Marzena Wątek $\mathbb{B}^{75,76} \cdot$ Matteo Pelosini $^{77}$. Elzbieta Iskierka-Jażdżewska ${ }^{78} \cdot$ Norbert Grząśko $^{79}$ - Joaquin Martinez-Lopez ${ }^{80} \cdot$ Andrés Jerez $^{81} \cdot$ Daniele Campa $^{82}$. Gabriele Buda ${ }^{76}$ - Fabienne Lesueur $\mathbb{D}^{83}$. Marek Dudziński ${ }^{84}$ - Ramón García-Sanz $\mathbb{D}^{85}$ - Arnon Nagler ${ }^{86}$. Marcin Rymko ${ }^{87} \cdot$ Krzysztof Jamroziak $^{75}$ - Aleksandra Butrym ${ }^{88} \cdot$ Federico Canzian $^{89} \cdot$ Ofure Obazee $^{89}$. Björn Nilsson ${ }^{2} \cdot$ Robert J. Klein $\mathbb{1}^{90}$ - Steven M. Lipkin ${ }^{4}$ James D. McKay ${ }^{1} \cdot$ Charles Dumontet ${ }^{5,6,8,9}$

Received: 5 October 2018 / Revised: 4 February 2019 / Accepted: 8 February 2019 / Published online: 9 April 2019

(c) The Author(s) 2019. This article is published with open access

\section{To the Editor:}

Multiple myeloma (MM) is the third most common hematological malignancy, after Non-Hodgkin Lymphoma and Leukemia. MM is generally preceded by Monoclonal Gammopathy of Undetermined Significance (MGUS) [1], and epidemiological studies have identified older age, male gender, family history, and MGUS as risk factors for developing MM [2].

The somatic mutational landscape of sporadic MM has been increasingly investigated, aiming to identify recurrent

These authors contributed equally: James D. McKay, Charles Dumontet

Supplementary information The online version of this article (https:// doi.org/10.1038/s41375-019-0452-6) contains supplementary material, which is available to authorized users.

James D. McKay

mckayj@iarc.fr

Charles Dumontet

charles.dumontet@chu-lyon.fr

Extended author information available on the last page of the article genetic events involved in myelomagenesis. Whole exome and whole genome sequencing studies have shown that MM is a genetically heterogeneous disease that evolves through accumulation of both clonal and subclonal driver mutations [3] and identified recurrently somatically mutated genes, including KRAS, NRAS, FAM46C, TP53, DIS3, BRAF, TRAF3, CYLD, RBI and PRDM1 [3-5].

Despite the fact that family-based studies have provided data consistent with an inherited genetic susceptibility to MM compatible with Mendelian transmission [6], the molecular basis of inherited MM predisposition is only partly understood. Genome-Wide Association (GWAS) studies have identified and validated 23 loci significantly associated with an increased risk of developing MM that explain $~ 16 \%$ of heritability [7] and only a subset of familial cases are thought to have a polygenic background [8]. Recent studies have identified rare germline variants predisposing to $\mathrm{MM}$ in KDM1A [9], ARIDIA and USP45 [10], and the implementation of nextgeneration sequencing technology will allow the characterization of more such rare variants.

In this study, we sought to explore the involvement of rare germline genetic variants in susceptibility to MM. 
Within our discovery cohort of peripheral blood samples (see Supplementary Methods) from 66 individuals from 23 unrelated families analyzed by WES, DIS3 (NM_014953) was the only gene in which putative loss-of-function variants were observed in at least two families. An additional cohort of 937 individuals (148 MM, 139 MGUS, 642 unaffected relatives and eight individuals with another hematological condition) from 154 unrelated families (including the individuals in the discovery cohort) were screened for germline variants in DIS3 using targeted sequencing (Supplementary Table S1). In total, we detected DIS3 germline putative lossof-function variants in four unrelated families. The DIS3 genotypes for the identified variants were concordant between WES and targeted sequencing (where available) and independently confirmed by Sanger sequencing on DNA extracted from uncultured whole blood. The variant allele frequencies (VAF) were close to $50 \%$, as expected of a germline variant (Supplementary figure $\mathrm{S} 1$ ).

The DIS3 gene, located in 13q22.1, encodes for the catalytic subunit of the human exosome complex, and is recurrently somatically mutated in $\mathrm{MM}$ patients [4, 5, 11, 12]. The somatic variants are predominantly missense variants localized in the RNB domain mainly abolishing the exoribonucleolytic activity [4, 13], and are often accompanied by $\mathrm{LOH}$ or biallelic inactivation due to $13 \mathrm{q} 14$ deletion, implying a tumor suppressor role for DIS3 in MM $[5,12,13]$.

The first DIS3 variant, observed in 2 affected siblings (1 MGUS and 1 MM case) from family B (Fig. 1a), was located in the splice donor site of exon 13 (c.1755+1G>T; chr13: 73,345,041; GRCh37/hg19, rs769194741) (Supplementary Figure S1a). It is predicted to abolish the splice donor site and cause skipping of exon 13, introducing a premature termination codon (p.Arg557Argfs*3) and result in a truncated DIS3 protein that lacks part of the exonucleolytic active RNB and S1 domains (Fig. 1b, c). The presence of this variant in two siblings, implying Mendelian segregation, is consistent with a germline, rather than somatic, origin. We investigated whether a DIS3 transcript from the variant allele is generated but is subsequently eliminated by Nonsense Mediated Decay (NMD) by incubating Lymphoblastoid Cell Lines (LCLs) derived from the two c.1755+1G>T allele carriers with and without puromycin, which suppresses NMD. The mRNA transcript corresponding to the variant allele was clearly present in LCLs treated with puromycin in both carriers, whereas not detectable in untreated LCLs (Fig. 2a), consistent with the variant allele being transcribed but subsequently degraded via the NMD pathway. In line with this observation, analysis of DIS3 mRNA expression by qRTPCR showed an average $50 \%$ reduced expression in the c. $1755+1 \mathrm{G}>\mathrm{T}$ carriers (range $40.7-61.4 \%$ ) as compared to non-carriers (Fig. 2b). A second splicing variant (c.1883 $+1 \mathrm{G}>\mathrm{C}$; chr13: 73,342,922; GRCh37/hg19) located in the splice donor site of exon 14 within the RNB domain was identified in a MM case from family D (Fig. 1a, b, Supplementary figure S1c). However, the individual's mother (Q59), affected with amyloidosis, did not carry the variant, implying that MM in the allele carriers' maternal uncles is unlikely to be explained by this DIS3 variant. Whether the mRNA transcript encoded by this germline variant undergoes NMD could not be explored due to lack of appropriate material (LCLs, RNA).

A third DIS3 variant disrupting the wild-type termination codon (stop-loss) (c.2875T>C; p.*959Gln; chr13:73,333,935; GRCh37/hg19, rs141067458) (Fig. 1b, Supplementary Figure S1b) was identified in two unrelated families (A and C, Fig. 1a). This variant is expected to result in a putative readthrough variant and a DIS3 protein with an additional 13 amino acids in the C-terminus (p.*959Glnext*14). It was detected in 3 out of 4 affected siblings (2 MGUS (M63, O53) and 1 MM case (O29)), as well as 5 unaffected relatives (N14, N13, L41, M33 M50) from family A. The Mendelian segregation of this variant in this pedigree is also consistent with germline origin. An additional $\mathrm{MM}$ case from family $\mathrm{C}$ carried the variant, while we were unable to assess the other MM-afflicted family member (Fig. 1a). As expected of a stop-loss variant, NMD was not observed (data not shown), and gene expression analysis showed no effect on DIS3 mRNA levels (Fig. 2b). However, western blot analysis demonstrated that DIS3 protein levels were markedly lower $(\sim 50 \%)$ in the p.*959Glnext*14 carrier (O53, family A) compared to non-carriers (Fig. 2b, c).

Next, we sought to determine if rare, putative deleterious variants in DIS3 were more frequent in an independent series of MM cases compared to unaffected individuals. We performed mutation burden tests between $781 \mathrm{MM}$ cases and 3534 controls from the MMRF CoMMpass Study with WES data available. After testing for systemic bias in this dataset (see Supplementary Methods, Supplementary Figure S2), we undertook a burden test for association between functional DIS3 variants and MM. DIS3 putative functional variants (truncating and likely deleterious missense variants, see Supplementary Methods) were more frequent among MM patients $(30 / 781)$ than controls $(72 / 3534)(\mathrm{OR}=1.9295 \%$ CI:1.25-2.96, $p=0.001)$. Although the p.*959Glnext*14 stop-loss variant was recurrently found in 10/781 MM cases and 15/3534 controls $(\mathrm{OR}=3.0795 \% \mathrm{CI}: 1.38$ to $6.87, p=$ $0.0007)$, it did not entirely explain the excess of DIS3 variants among cases as there is evidence for association with other putative functional variants (Supplementary Figure S3a). We additionally genotyped the p.*959Glnext*14 stop-loss variant in an independent series of sporadic MM cases and controls from the IMMEnSE Consortium. While this variant was very rare in this series (8/3020 MM cases relative to 3/1786 controls), there was a consistent but non-significant association between this variant and $\mathrm{MM}(\mathrm{OR}=3.1595 \% \mathrm{CI}$ : 0.74 $13.43 p=0.122$ ). 


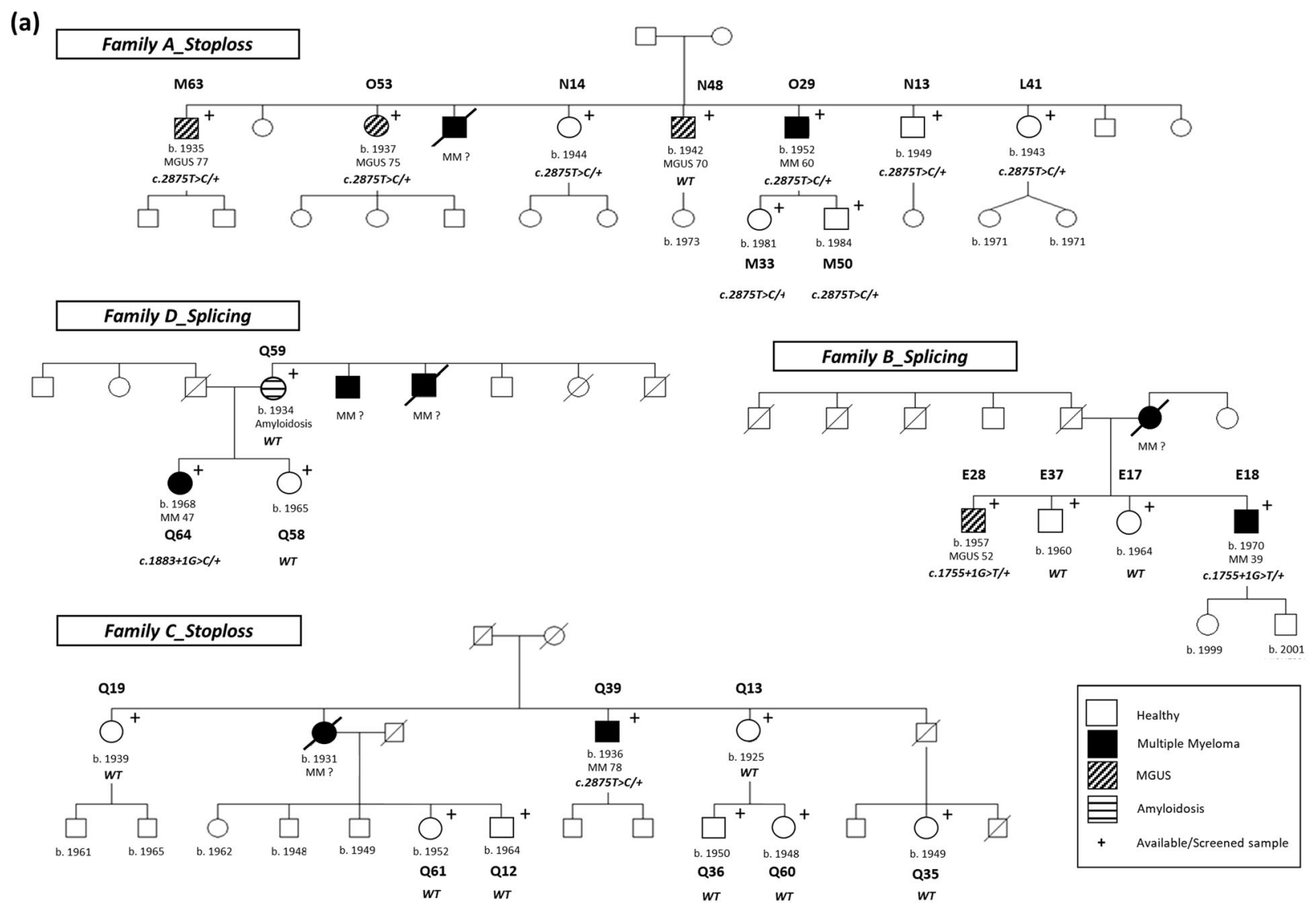

(b) Germline (our study \& MMRF COMMPASS)

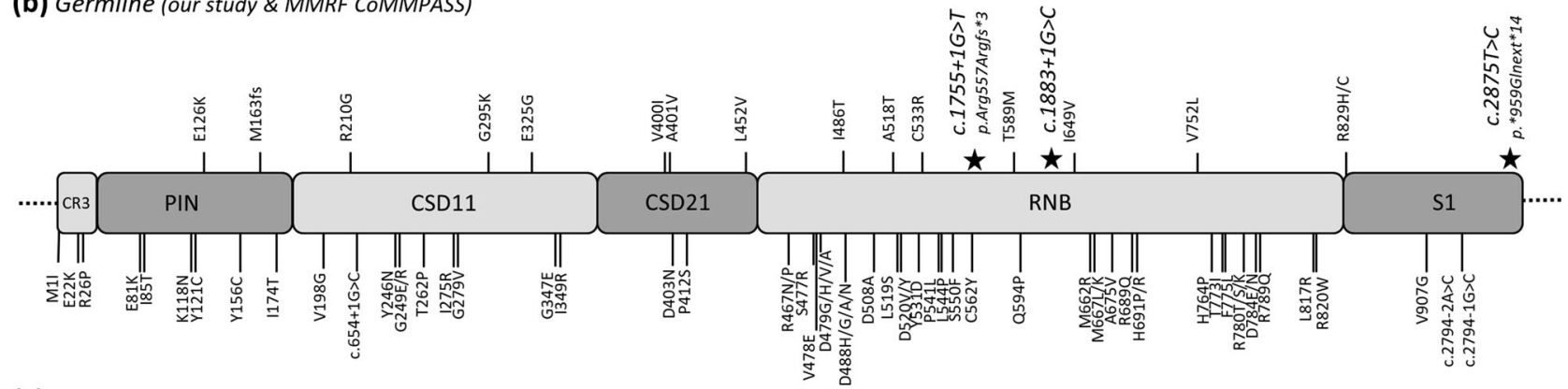

(c) Somatic (MMRF COMMPASS)

Fig. 1 DIS3 variants in MM cases. a Pedigrees from families carrying a germline DIS3 variant. Available samples for screening are marked with a "+" symbol. Families A and C carry the p.*959Glnext*14 (c.2875T $>$ C) stop-loss variant. Family B carries the c.1755+1G $>$ T splicing variant and family $\mathrm{D}$ carries the $\mathrm{c} .1883+1 \mathrm{G}>\mathrm{C}$ splicing variant. The genotype of all screened individuals is shown on each pedigree. WT: wild type. b, c Schematic representation of identified germline and somatic variants in the distinct DIS3 protein domains. b Germline variants were identified through WES and targeted

To explore the functional consequence of germline DIS3 variants, we compared MM tumor transcriptomes from patients harboring germline $(n=21)$ and somatic $(n=$ 96) DIS3 putative functional variants to non-carriers $(n=$ 655). Differential expression analyses showed an enrichment of pathways associated with global ncRNA processing and translational termination in germline DIS3 carriers including resequencing in families with reoccurrence of MM/MGUS as well as in a collection of sporadic MM cases (MMRF CoMMpass Study). The DIS3 variants discussed in the present study are depicted with a star on the upper part of the figure. c Somatic DIS3 variants were identified in sporadic MM cases from the MMRF CoMMpass Study. We observe that in contrast to the clustering of somatic DIS3 missense variants in the RNB and PIN domains, germline variants are scattered throughout the gene and consist of splicing, stop-loss and missense variants

ncRNA processing, ncRNA metabolic process, translational termination, and RNA metabolism. Among somatic DIS3 carriers, significantly enriched pathways include interferon alpha/beta signalling, mRNA splicing, mRNA processing and transcription (Supplementary Figure S3b, Supplementary tables $\mathrm{S} 3$ and $\mathrm{S} 4 \mathrm{a}-\mathrm{d})$. These findings are consistent with the proposed DIS3 role in regulating mRNA processing [14] and 
(a)

WITHOUT PUROMYCIN

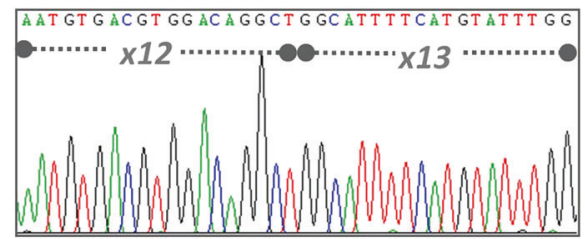

PELLSSNLCSLKCDVDRLAFSCIWEMNHNAEILKTKFTKSVINSKASLTYAEAQLRIDSA
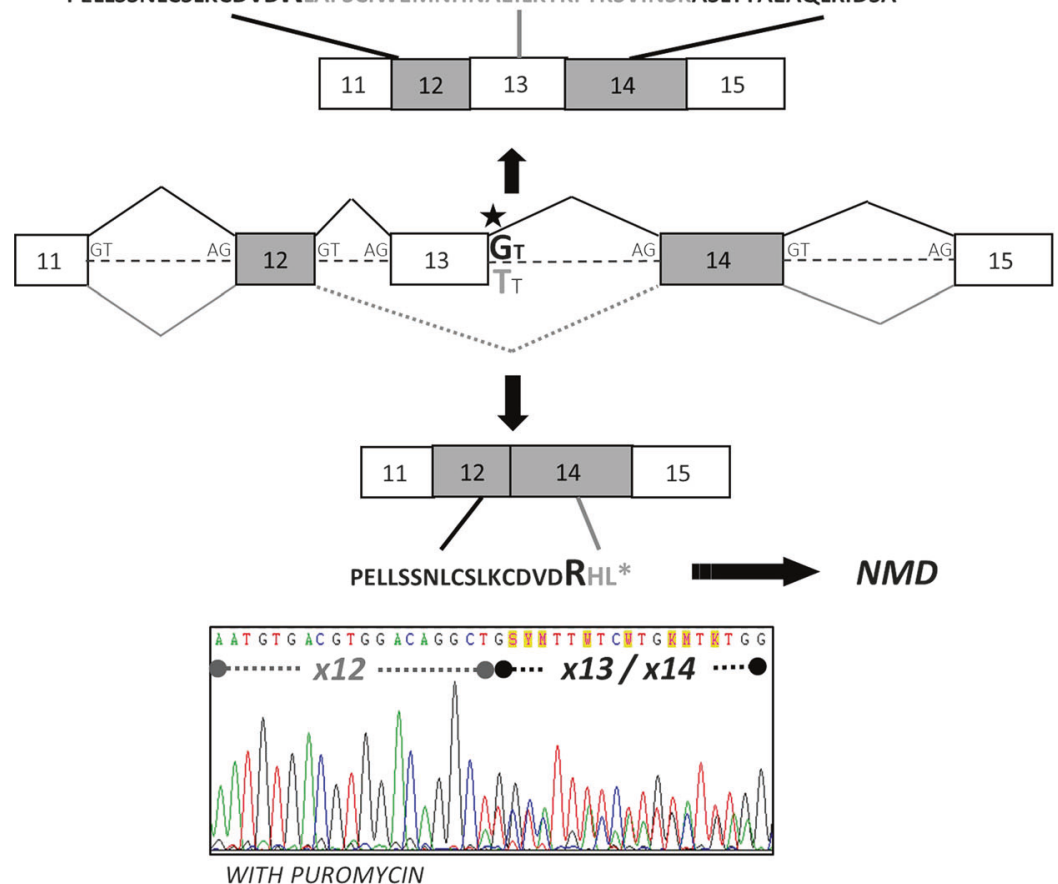

Fig. 2 DIS3 c. $1755+1 \mathrm{G}>\mathrm{T}$ splicing variant results in nonsensemediated mRNA decay (NMD) and affects mRNA expression, while the c. $2875 \mathrm{C}>\mathrm{T}$ (p.*959Glnext*14) stop-loss variant affects protein levels. a LCLs from patients E18 and E28 (not shown) carrying the c. $1755+1 \mathrm{G}>\mathrm{T}$ splicing variant were cultured with and without puromycin. The chromatogram from treated cells (with puromycin) showed a mixture of the wild-type and mutant transcript lacking exon 13, which was not detected in the non-treated cells (without puromycin). Thus, the mutant transcript is degraded by NMD. b Box plot

more specifically mRNA decay, gene expression and small RNA processing [15]. We also observed that, several longintergenic non-protein coding RNAs, non-coding and antisense RNAs were significantly enriched among DIS3 carriers (Supplementary table S5a, b) supporting previous studies that demonstrate an accumulation of transcripts from non-protein coding regions, snoRNA precursors and certain lncRNAs in DIS3 mutant cells, along a general deregulation of mRNA levels probably due to the sequestration of transcriptional factors from the accumulated nuclear RNAs [16].

To our knowledge, this is the first observation of germline DIS3 likely deleterious variants in familial $\mathrm{MM}$ and our results suggest that the involvement of DIS3 in MM etiology may extend beyond somatic alterations to germline susceptibility. We reported rare germline DIS3 variants in $~ 2.6 \%$ of our cohort of families with multiple cases of MM and MGUS (b)

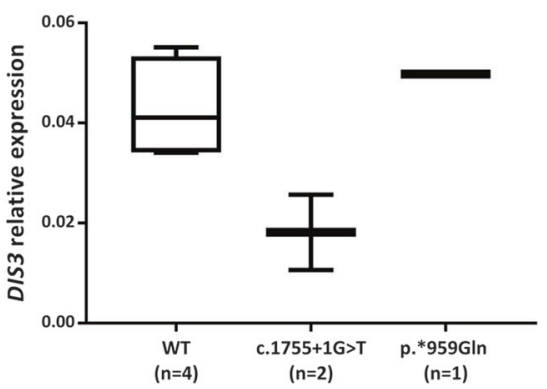

(c)

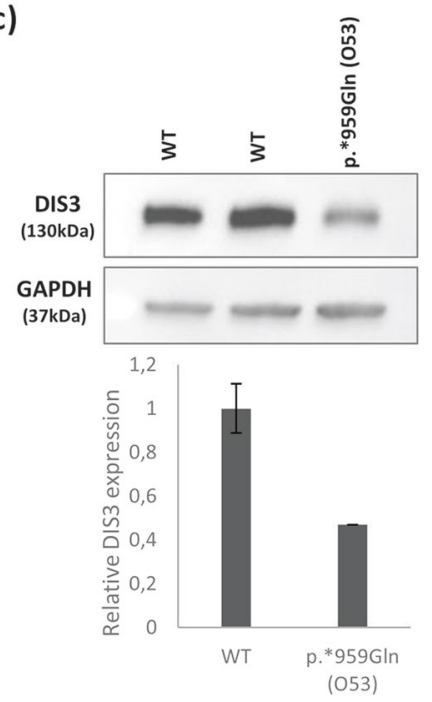

representing the relative DIS3 mRNA expression in c. $1775+1 \mathrm{G}>\mathrm{A}$ $(\mathrm{n}=2)$ and $\mathrm{p} .{ }^{*} 959 \mathrm{Glnext} * 14(n=1)$ carriers compared to non-carriers $(n=4)$. All reactions were performed in triplicates. c Western blot with an anti-DIS3 antibody was performed in LCLs from one p. *959Glnext*14 carrier and two wild-type individuals (anti-GAPDH antibody as internal control). The relative DIS3 expression in the p. *959GInext*14 carrier was reduced by $50 \%$ compared to non-carriers, suggesting that the mutant allele is translated but degraded shortly after

(4/154). The germline variants described here are predicted to have loss-of-function impact on DIS3. Consistent with this, the $1755+1 \mathrm{G}>\mathrm{T}$ (rs769194741) splicing variant induces NMD and results in reduced DIS3 mRNA expression, supporting the proposal that DIS3 is acting as a tumor suppressor gene in MM [13]. Moreover, the c.2875T >C (rs141067458) stop-loss variant (p.*959Glnext*14) results in reduced DIS3 protein expression suggesting that the mutant allele is translated but degraded shortly after. Notably, in contrast to the clustering of somatic DIS3 mutations in the PIN and RNB domains, germline variants identified both in familial and sporadic MM cases are scattered throughout the gene (Fig. 1b, c). Despite the fact that these variants do not segregate perfectly with $\mathrm{MM}$ in the identified families and the rarity of DIS3 germline likely deleterious variants limits our statistical power, the subsequent mutation burden and transcriptome 
analyses provided supportive data towards DIS3 acting as an "intermediate-risk" MM susceptibility gene.

Acknowledgements This work was supported by the French National Cancer Institute (INCA) and the Fondation Française pour la Recherche contre le Myélome et les Gammapathies (FFMRG), the Intergroupe Francophone du Myélome (IFM), NCI R01 NCI CA167824 and a generous donation from Matthew Bell. This work was supported in part through the computational resources and staff expertise provided by Scientific Computing at the Icahn School of Medicine at Mount Sinai. Research reported in this paper was supported by the Office of Research Infrastructure of the National Institutes of Health under award number S10OD018522. The content is solely the responsibility of the authors and does not necessarily represent the official views of the National Institutes of Health. The authors thank the Association des Malades du Myélome Multiple (AF3M) for their continued support and participation. Where authors are identified as personnel of the International Agency for Research on Cancer / World Health Organization, the authors alone are responsible for the views expressed in this article and they do not necessarily represent the decisions, policy or views of the International Agency for Research on Cancer / World Health Organization.

\section{Compliance with ethical standards}

Conflict of interest The authors declare that they have no conflict of interest.

Publisher's note: Springer Nature remains neutral with regard to jurisdictional claims in published maps and institutional affiliations.

Open Access This article is licensed under a Creative Commons Attribution 4.0 International License, which permits use, sharing, adaptation, distribution and reproduction in any medium or format, as long as you give appropriate credit to the original author(s) and the source, provide a link to the Creative Commons license, and indicate if changes were made. The images or other third party material in this article are included in the article's Creative Commons license, unless indicated otherwise in a credit line to the material. If material is not included in the article's Creative Commons license and your intended use is not permitted by statutory regulation or exceeds the permitted use, you will need to obtain permission directly from the copyright holder. To view a copy of this license, visit http://creativecommons. org/licenses/by/4.0/.

\section{References}

1. Weiss BM, Abadie J, Verma P, Howard RS, Kuehl WM. A monoclonal gammopathy precedes multiple myeloma in most patients. Blood. 2009;113:5418-22.
2. Morgan GJ, Davies FE, Linet M. Myeloma aetiology and epidemiology. Biomed Pharmacother. 2002;56:223-34.

3. Bolli N, Avet-Loiseau H, Wedge DC, Van Loo P, Alexandrov LB, Martincorena I, et al. Heterogeneity of genomic evolution and mutational profiles in multiple myeloma. Nat Commun. 2014; 5:2997.

4. Chapman MA, Lawrence MS, Keats JJ, Cibulskis K, Sougnez C, Schinzel AC, et al. Initial genome sequencing and analysis of multiple myeloma. Nature. 2013;471:467-72.

5. Lohr JG, Stojanov P, Carter SL, Cruz-Gordillo P, Lawrence MS, Auclair D, et al. Widespread genetic heterogeneity in multiple myeloma: implications for targeted therapy. Cancer Cell. 2014; 25:91-101.

6. Morgan GJ, Johnson DC, Weinhold N, Goldschmidt H, Landgren O, Lynch HT, et al. Inherited genetic susceptibility to multiple myeloma. Leukemia. 2014;28:518-24.

7. Went M, Sud A, Försti A, Halvarsson B-M, Weinhold N, Kimber S, et al. Identification of multiple risk loci and regulatory mechanisms influencing susceptibility to multiple myeloma. Nat Commun. 2018;9:3707.

8. Halvarsson B-M, Wihlborg A-K, Ali M, Lemonakis K, Johnsson E, Niroula A, et al. Direct evidence for a polygenic etiology in familial multiple myeloma. Blood Adv. 2017;1:619-23.

9. Wei X, Calvo-Vidal MN, Chen S, Wu G, Revuelta MV, Sun J, et al. Germline mutations in lysine specific demethylase 1 (LSD1/ KDM1A) confer susceptibility to multiple myeloma. Cancer research 2018. https://doi.org/10.1158/0008-5472.CAN-17-1900.

10. Waller RG, Darlington TM, Wei X, Madsen MJ, Thomas A, Curtin K, et al. Novel pedigree analysis implicates DNA repair and chromatin remodeling in multiple myeloma risk. PLoS Genet. 2018;14:e1007111.

11. Weißbach S, Langer C, Puppe B, Nedeva T, Bach E, Kull M, et al. The molecular spectrum and clinical impact of DIS3 mutations in multiple myeloma. Br J Haematol. 2015;169:57-70.

12. Walker BA, Mavrommatis K, Wardell CP, Ashby TC, Bauer M, Davies FE, et al. Identification of novel mutational drivers reveals oncogene dependencies in multiple myeloma. Blood 2018;132:587-97.

13. Lionetti M, Barbieri M, Todoerti K, Agnelli L, Fabris S, Tonon G, et al. A compendium of DIS3 mutations and associated transcriptional signatures in plasma cell dyscrasias. Oncotarget. 2015; 6. https://doi.org/10.18632/oncotarget.4674.

14. Dziembowski A, Lorentzen E, Conti E, Séraphin B. A single subunit, Dis3, is essentially responsible for yeast exosome core activity. Nat Struct Mol Biol. 2007;14:15-22.

15. Robinson S, Oliver A, Chevassut T, Newbury S. The 3' to 5' exoribonuclease DIS3: from structure and mechanisms to biological functions and role in human disease. Biomolecules. 2015;5:1515-39.

16. Szczepińska T, Kalisiak K, Tomecki R, Labno A, Borowski LS, Kulinski TM, et al. DIS3 shapes the RNA polymerase II transcriptome in humans by degrading a variety of unwanted transcripts. Genome Res. 2015;25:1622-33.

Maroulio Pertesi $^{1,2} \cdot$ Maxime Vallée $^{1} \cdot$ Xiaomu Wei $^{3} \cdot$ Maria V. Revuelta $\mathbb{D}^{4} \cdot$ Perrine Galia $^{5,6} \cdot$ Delphine Demangel $^{5,6}$. Javier Oliver ${ }^{1,7} \cdot$ Matthieu Foll $^{1} \cdot$ Siwei Chen $^{3} \cdot$ Emeline Perrial $^{8,9} \cdot$ Laurent Garderet $^{10,11,12} \cdot$ Jill Corre $^{13}$. Xavier Leleu ${ }^{14}$ - Eileen M. Boyle ${ }^{15}$. Olivier Decaux ${ }^{16,17,18}$. Philippe Rodon ${ }^{19}$. Brigitte Kolb $^{20}$ - Borhane Slama ${ }^{21}$. Philippe Mineur $^{22}$. Eric Voog ${ }^{23}$ - Catherine Le Bris ${ }^{24}$ - Jean Fontan ${ }^{25}$. Michel Maigre ${ }^{26}$ - Marie Beaumont ${ }^{27}$. Isabelle Azais ${ }^{28}$ - Hagay Sobol ${ }^{29}$ - Marguerite Vignon ${ }^{30}$ - Bruno Royer ${ }^{30}$ - Aurore Perrot ${ }^{31}$ - Jean-Gabriel Fuzibet ${ }^{32}$. Véronique Dorvaux ${ }^{33} \cdot$ Bruno Anglaret $^{34}$ - Pascale Cony-Makhoul ${ }^{35}$. Christian Berthou ${ }^{36}$. Florence Desquesnes ${ }^{37}$. Brigitte Pegourie $^{38} \cdot$ Serge Leyvraz $^{39} \cdot$ Laurent Mosser $^{40} \cdot$ Nicole Frenkiel $^{41} \cdot$ Karine Augeul-Meunier $^{42}$. Isabelle Leduc ${ }^{43}$. Cécile Leyronnas ${ }^{44}$. Laurent Voillat ${ }^{45} \cdot$ Philippe Casassus $^{46} \cdot$ Claire Mathiot $^{47} \cdot$ Nathalie Cheron $^{48}$. Etienne Paubelle ${ }^{49} \cdot$ Philippe Moreau $^{50} \cdot$ Yves-Jean Bignon ${ }^{51} \cdot$ Bertrand Joly $^{52} \cdot$ Pascal Bourquard $^{53} \cdot$ Denis Caillot $^{54}$. 
Hervé Naman ${ }^{55} \cdot$ Sophie Rigaudeau ${ }^{56} \cdot$ Gérald Marit $^{57} \cdot$ Margaret Macro $^{58} \cdot$ Isabelle Lambrecht $^{59}$.

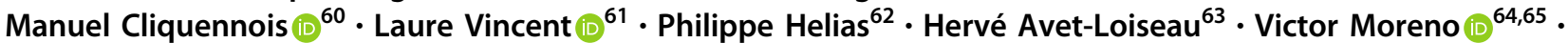
Rui Manuel Reis ${ }^{66,67} \cdot J^{\prime} u d i t$ Varkonyi ${ }^{68} \cdot$ Marcin Kruszewski $^{69} \cdot$ Annette Juul Vangsted $^{70} \cdot$ Artur Jurczyszyn $^{71}$. Jan Maciej Zaucha ${ }^{72}$. Juan Sainz (D) $^{73} \cdot$ Malgorzata Krawczyk-Kulis $^{74}$ - Marzena Wątek (iD ${ }^{75,76} \cdot$ Matteo Pelosini $^{77}$. Elzbieta Iskierka-Jażdżewska ${ }^{78}$ • Norbert Grząśko ${ }^{79} \cdot J_{0}$ oaquin Martinez-Lopez ${ }^{80} \cdot$ Andrés Jerez $^{81} \cdot$ Daniele Campa $^{82}$. Gabriele Buda $^{76}$ - Fabienne Lesueur ${ }^{83} \cdot$ Marek Dudziński $^{84} \cdot$ Ramón García-Sanz $^{85} \cdot$ Arnon Nagler $^{86}$. Marcin Rymko ${ }^{87}$. Krzysztof Jamroziak ${ }^{75}$ - Aleksandra Butrym ${ }^{88} \cdot$ Federico Canzian $^{89} \cdot$ Ofure Obazee $^{89}$. Björn Nilsson ${ }^{2} \cdot$ Robert J. Klein ${ }^{90}$ - Steven M. Lipkin ${ }^{4} \cdot$ James D. McKay ${ }^{1} \cdot$ Charles Dumontet $^{5,6,8,9}$

1 Genetic Cancer Susceptibility, International Agency for Research on Cancer, Lyon, France

2 Department of Laboratory Medicine, Division of Hematology and Transfusion medicine, Lund University, Lund, Sweden

3 Biological Statistics and Computational Biology, Cornell University, Ithaca, NY, USA

4 Medicine, Weill Cornell Medical College, New York, NY, USA

5 ProfilExpert, Lyon, France

6 Hospices Civils de Lyon, Lyon, France

7 Medical Oncology Service, Hospitales Universitarios Regional y Virgen de la Victoria; Institute of Biomedical Research in Malaga (IBIMA), CIMES, University of Málaga, Málaga, Spain

8 INSERM 1052, CNRS 5286, CRCL, Lyon, France

9 University of Lyon, Lyon, France

10 INSERM, UMR_S 938, Paris, France

11 AP-HP, Hôpital Saint Antoine, Departement d'hematologie et de therapie cellulaire, Paris, France

12 Sorbonne Universites, UPMC Univ Paris 06, UMR_S 938, Paris, France

13 IUC-Oncopole and CRCT INSERM U1037, Toulouse, France

14 Inserm CIC 1402 \& Service d'Hématologie et Thérapie Cellulaire, CHU La Miletrie, Poitiers, France

15 Hôpital Claude Huriez, CHRU, Lille, France

16 Service de Medecine Interne, CHU Rennes, Rennes, France

17 Faculte de Medecine, Universite de Rennes 1, Rennes, France

18 INSERM UMR U1236, Rennes, France

19 Unite d'Hematologie et d'Oncologie, Centre Hospitalier, Perigueux, France

20 Hematologie Clinique, CHU de Reims, Reims, France

21 Service d'Onco hematologie, $\mathrm{CH}$ Avignon, Avignon, France

22 Hematologie et pathologies de la coagulation, Grand Hôpital de Charleroi, Charleroi, Belgium

23 Centre Jean Bernard, Institut Inter-regional de Cancerologie, Le Mans, France

24 Service post urgences, CHU de FORT DE FRANCE, pôle RASSUR, Martinique, France

25 Hopital Jean Minjoz, CHRU Besançon, Besançon, France
26 Service d'Hemato-Oncologie, CHU Chartres, Chartres, France

27 Hematologie clinique et therapie cellulaire, CHU Amiens, Amiens, France

28 Service de rhumatologie, CHU Poitiers, Poitiers, France

29 Cancer Genetics Department, Paoli-Calmettes Institute, AixMarseille University, Marseille, France

30 Service d'Immuno-hematologie, Hôpital Saint Louis, Paris, France

31 Service d'Hematologie, CHU de Nancy, Universite de Lorraine, Vandoeuvre les Nancy, Nancy, France

32 Internal Medicine Department, Archet Hospital, CHU Nice, Nice, France

33 Service d'Hematologie, CHR Mercy, Metz, France

34 Unite d'Hematologie, $\mathrm{CH}$ Valence, Valence, France

35 Service d'Hematologie, Centre Hospitalier Annecy Genevois, Epagny Metz-Tessy, France

36 Service d'Hematologie, CHU de Brest, Brest, France

37 Haematology Department, CHU UCL Namur, Yvoir, Belgium

38 Hematologie clinique, CHU de Grenoble, La Tronche, France

39 Departement d'oncologie, CHUV, Lausanne, Switzerland

40 Unite d'oncologie medicale, Pôle medical 2, Hôpital Jacques Puel, Rodez, France

41 CH Poissy, Saint-Germain-en-Laye, France

42 Service Hematologie, Institut de Cancerologie Lucien Neuwirth, Saint-Priest-en-Jarez, France

43 Hematologie, CHG Abbeville, Abbeville, France

44 Institut Daniel Hollard, Groupe Hospitalier Mutualiste de Grenoble, Grenoble, France

45 Service hemato/oncologie, CH William Morey, Chalon sur Saône, France

46 Hematologie clinique, Hôpital Avicenne, Bobigny, France

47 Intergroupe Francophone du Myelome (IFM), Bobigny, France

48 Service Hematologie, CH Bligny, Briis-sous-Forges, France

49 Service Hematologie, CH Lyon Sud, Pierre Benite, France

50 Service Hematologie, CHU Nantes, Nantes, France

51 Laboratoire de Biologie Medicale OncoGènAuvergne; 
Departement d'oncogenetique, UMR INSERM 1240, Centre Jean Perrin, Clermont-Ferrand, France

52 Service d'hematologie clinique, Pôle medecine de specialite, Centre Hospitalier Sud Francilien (CHSF), Corbeil-

Essonnes, France

53 Hematologie Clinique, CHU Nîmes, Nîmes, France

54 Hematologie Clinique, CHU Dijon, Dijon, France

55 Hematologie - Oncologie medicale, Centre Azureen de Cancerologie, Mougins, France

56 Service d'Hematologie et d'Oncologie, CHU de Versailles, Le Chesnay, France

57 INSERM U1035, Universite de Bordeaux, Bordeaux, France

58 Hematologie Clinique, IHBN-CHU CAEN (University Hospital), Caen, France

59 Rheumatology Department, Maison Blanche Hospital, Reims University Hospitals, Reims, France

60 Unite d'Hematologie clinique, Groupement des hôpitaux de l'Institut Catholique (GHICL), Universite Catholique de Lille, Lille, France

61 Departement d'hematologie clinique, CHU de Montpellier, Montpellier, France

62 Service d'Oncologie medicale, CHU de La Guadeloupe, Pointe-aPitre, Guadeloupe

63 Laboratory for Genomics in Myeloma, Institut Universitaire du Cancer and University Hospital, Centre de Recherche en Cancerologie de Toulouse, Toulouse, France

64 CIBER Epidemiología y Salud Pública (CIBERESP), Madrid, Spain

65 Unit of Biomarkers and Susceptibility, Cancer Prevention and Control Program, IDIBELL, Catalan Institute of Oncology; Department of Clinical Sciences, Faculty of Medicine, University of Barcelona, Barcelona, Spain

66 Life and Health Sciences Research Institute (ICVS), School of Health Sciences, University of Minho, Braga, Portugal; ICVS/ 3B's-PT Government Associate Laboratory, Braga/ Guimarães, Portugal

67 Molecular Oncology Research Center, Barretos Cancer Hospital, Barretos, São Paulo, Brazil

68 3rd Department of Internal Medicine, Semmelweis University, Budapest, Hungary

69 Department of Hematology, University Hospital, Bydgoszcz, Poland

70 Department of Haematology, Rigshospitalet, Copenhagen University, Copenhagen, Denmark
71 Jagiellonian University Medical College, Department of Hematology, Cracow, Poland

72 Gdynia Oncology Center, Gdynia and Department of Oncological Propedeutics, Medical University of Gdańsk, Gdańsk, Poland

73 Genomic Oncology Area, GENYO. Centre for Genomics and Oncological Research: Pfizer/University of Granada/Andalusian Regional Government, PTS Granada, Granada, Spain

74 Department of Bone Marrow Transplantation and HematologyOncology M. Sklodowska-Curie Memorial Cancer Center and Institute of Oncology Gliwice Branch, Gliwice, Poland

75 Department of Hematology, Institute of Hematology and Transfusion Medicine, Warsaw, Poland

76 Holycross Cancer Center of Kielce, Hematology Clinic, Kielce, Poland

77 Department of Oncology, Transplants and Advanced Technologies, Section of Hematology, Pisa University Hospital, Pisa, Italy

78 Department of Hematology, Medical University of Lodz, Łódź, Poland

79 Department of Experimental Hemato-oncology, Medical University of Lubli, Poland; Department of Hematology, St. John's Cancer Centre, Polish Myeloma Study Group, Lublin, Poland

80 Hematology Department, Hospital 12 de Octubre, Universidad Complutense; CNIO, Madrid, Spain

81 Hematology and Medical Oncology Department, Hospital Morales Meseguer, IMIB, Murcia, Spain

82 Department of Biology, University of Pisa, Pisa, Italy

83 Inserm U900, Institut Curie, PSL Research University, Mines ParisTech, Paris, France

84 Teaching Hospital No1, Hematology Dept, Rzeszow, Poland

85 Hematology Department, University Hospital of Salamanca, IBSAL, Salamanca, Spain

86 Hematology Division, Chaim Sheba Medical Center, Tel Hashomer, Israel

87 Department of Hematology, Copernicus Hospital, Torun, Poland

88 Wroclaw Medical University, Wroclaw, Poland

89 Genomic Epidemiology Group, German Cancer Research Center (DKFZ), Heidelberg, Germany

90 Department of Genetics and Genomic Sciences and Icahn Institute for Genomics and Multiscale Biology, Icahn School of Medicine at Mount Sinai, New York, NY, USA 